\title{
Nonlinear stability of continuously stratified quasi-geostrophic flow
}

Article

Published Version

Yongming, L., Mu, M. and Shepherd, T. G. (1996) Nonlinear stability of continuously stratified quasi-geostrophic flow. Journal Of Fluid Mechanics, 325. pp. 419-439. ISSN 00221120 doi: https://doi.org/10.1017/S002211209600818X Available at https://centaur.reading.ac.uk/32871/

It is advisable to refer to the publisher's version if you intend to cite from the work. See Guidance on citing.

Published version at: http://dx.doi.org/10.1017/S002211209600818X

To link to this article DOI: http://dx.doi.org/10.1017/S002211209600818X

Publisher: Cambridge University Press

All outputs in CentAUR are protected by Intellectual Property Rights law, including copyright law. Copyright and IPR is retained by the creators or other copyright holders. Terms and conditions for use of this material are defined in the End User Agreement.

\section{www.reading.ac.uk/centaur}

\section{CentAUR}

Central Archive at the University of Reading

Reading's research outputs online 


\title{
Nonlinear stability of continuously stratified quasi-geostrophic flow
}

\author{
By LIU YONGMING ${ }^{1}$, MU MU $\mathrm{U}^{2}$ \\ AND THEODORE G. SHEPHER D \\ ${ }^{1}$ Institute of Mathematics, Anhui University, Hefei 230039, China \\ ${ }^{2}$ LASG, Institute of Atmospheric Physics, Chinese Academy of Sciences, Beijing 100029, China \\ ${ }^{3}$ Department of Physics, University of Toronto, Toronto M5S 1A7, Canada
}

(Received 26 April 1996)

\begin{abstract}
Nonlinear stability theorems analogous to Arnol'd's second stability theorem are established for continuously stratified quasi-geostrophic flow with general nonlinear boundary conditions in a vertically and horizontally confined domain. Both the standard quasi-geostrophic model and the modified quasi-geostrophic model (incorporating effects of hydrostatic compressibility) are treated. The results establish explicit upper bounds on the disturbance energy, the disturbance potential enstrophy, and the disturbance available potential energy on the horizontal boundaries, in terms of the initial disturbance fields. Nonlinear stability in the sense of Liapunov is also established.
\end{abstract}

\section{Introduction}

Building on the variational stability method of Fjørtoft (1950), Arnol'd (1966) established two exact, nonlinear stability theorems for the two-dimensional Euler equations. It was subsequently realized that these stability theorems arise from fundamental, symmetry-related aspects of the dynamics, which are most concisely expressed in terms of the Hamiltonian structure of the Euler equations (Holm et al. 1985; McIntyre \& Shepherd 1987; Shepherd 1990), and which may therefore generalize to other fluid dynamical models possessing Hamiltonian structure. This insight led to a proliferation of interest over the past decade in the establishment and application of nonlinear stability theorems, analogous to those of Arnol'd (1966), for various conservative fluid dynamical models (e.g. Holm et al. 1985; Swaters 1986; McIntyre \& Shepherd 1987; Szeri \& Holmes 1988; Shepherd 1988a, 1989; Mu \& Zeng 1991; Cho, Shepherd \& Vladimirov 1993; Bowman \& Shepherd 1995). This activity has been perhaps most fruitful in the field of atmospheric and oceanic dynamics. It turns out that virtually all of the classical inviscid linear stability theorems (e.g. static stability, symmetric stability, Rayleigh's inflection-point and centrifugalstability theorems, and the Fjørtoft-Pedlosky and Charney-Stern theorems) may be understood in this broader context, and may furthermore be extended to finite amplitude.

Arnol'd's derivation relies on the construction of a conserved functional, known variously as the 'energy-Casimir invariant' or 'pseudoenergy' (possibly augmented by inclusion of the momentum or impulse). The first theorem corresponds to cases where the conserved functional is positive definite; the second theorem to cases where 
it is negative definite. While the generalization of Arnol'd's first theorem to other fluid dynamical models is relatively straightforward, the generalization of the second theorem has, in contrast, proven to be far more difficult. Yet the second theorem is of considerable inherent interest, particularly in the context of large-scale atmospheric and oceanic dynamics where it is far more relevant, at least at face value, than is the first theorem (Andrews 1984).

The difficulties connected with Arnol'd's second theorem have to do with the necessary use of a Poincare inequality. This has two important consequences. First, any such stability theorem depends explicitly on the geometry of the flow domain. Second, the results depend critically on the presumed boundary conditions. Indeed, in his analysis Arnol'd (1966) restricted attention to isovortical disturbances, with zero disturbance circulation on the boundaries (see McIntyre \& Shepherd 1987, §6). In the case of two-dimensional Euler flow and layered quasi-geostrophic models, the difficulties have recently been overcome by $\mathrm{Mu} \&$ Shepherd (1994) and $\mathrm{Mu}$ et al. (1994), and nonlinear stability theorems analogous to Arnol'd's second theorem now exist for non-zero disturbance circulation. It should however be noted that those stability theorems are no longer strictly Liapunov; rather, they establish upper bounds on disturbance norms in terms of the initial disturbance fields, which go to zero uniformly as the initial disturbance goes to zero. This definition of nonlinear stability is, of course, still very useful for applications.

In the case of continuously stratified quasi-geostrophic flow, McIntyre \& Shepherd (1987) established an analogue of Arnol'd's second theorem for a vertically confined periodic zonal channel, but only under homogeneous boundary conditions: namely zero disturbance circulation on the sidewalls, and zero disturbance temperature on the horizontal boundaries (or lids). While dynamically consistent for basic states with isentropic horizontal boundaries, this assumption is severely restrictive for atmospheric and oceanic applications, where boundary temperature gradients are crucial to the dynamics of baroclinic instability and other phenomena. However, the problem of including inhomogeneous boundary conditions in this case is even more difficult than in the case of layered models, because the boundary condition on the horizontal surfaces is nonlinear.

Some progress on this problem has recently been made by Mu \& Wang (1992) and Mu \& Simon (1993), who were able to establish Poincaré inequalities that could be used in the context of inhomogeneous boundary conditions, and from this derive nonlinear stability theorems analogous to Arnol'd's second theorem. However, the strength of the resulting stability theorems depends on the tightness of the Poincaré inequality, and the Poincare inequalities used by $\mathrm{Mu} \&$ Wang (1992) and $\mathrm{Mu} \&$ Simon (1993) turn out to be far from optimal. Moreover, the bounds on disturbance norms derived in those studies are only implicit; this makes application of the results difficult, particularly in the case of Shepherd's (1988b; see also 1988a, 1989) method for obtaining saturation bounds for unstable flows. In addition, Liapunov stability was not established.

In this paper, we derive a Poincare inequality that is demonstrated to be optimal, and use it to prove nonlinear stability theorems analogous to Arnol'd's second theorem for continuously stratified quasi-geostrophic flow with general nonlinear boundary conditions in a vertically and horizontally confined domain. Both the standard quasigeostrophic model (e.g. Pedlosky 1987) and the modified quasi-geostrophic model of White (1977) are treated. We establish explicit upper bounds on the disturbance energy, the disturbance potential enstrophy, and the disturbance available potential energy on the horizontal boundaries, in terms of the initial disturbance fields, with 
the bounds going to zero uniformly as the initial disturbance goes to zero. Nonlinear stability in the sense of Liapunov is also established.

The plan of the paper is as follows. The governing equations are introduced in $\S 2$, and the disturbance problem described in $\$ 3$. The crucial Poincare inequality is established in $\$ 4$, and the resulting nonlinear stability theorem derived in $\S 5$. The case of basic states with uniform potential vorticity requires special treatment, which is provided in $\S 6$. In $\S 7$, the new stability theorems are shown to reduce to previous results in some special (restricted) cases. An example is considered in $\S 8$, and the paper concludes with a Discussion.

\section{Governing equations and integral invariants}

We consider the most general model of three-dimensional, continuously stratified, quasi-geostrophic flow on a beta-plane, due to White (1977). (This model is a generalization of the standard quasi-geostrophic model (e.g. Pedlosky 1987) that allows for effects of (hydrostatic) compressibility, which are important for large horizontal scales of motion.) The dynamics are governed by material conservation of potential vorticity $P$ at each vertical level $z$ :

$$
\frac{\mathrm{D} P}{\mathrm{D} t} \equiv P_{t}+\partial(\Phi, P)=0
$$

where $t$ is the time, $\Phi$ is the stream function, $\partial(F, G) \equiv F_{x} G_{y}-F_{y} G_{x}$ is the horizontal Jacobian operator, $x$ and $y$ are zonal and meridional coordinates, and

$$
P \equiv \nabla^{2} \Phi+\frac{1}{\rho}\left(r \Phi_{z}\right)_{z}+f+\beta y .
$$

Here $\rho=\rho(z)>0$ is the prescribed reference-state density; $f$ is the (constant) Coriolis parameter; $\beta$ is the (constant) planetary vorticity gradient; $r=r(z) \equiv \rho / S$, where $S \equiv N^{2}(z) / f^{2}>0$ is the prescribed reference-state static stability with $N^{2}(z)=$ $-g \rho_{z} / \rho-(g / c)^{2}$ the square of the buoyancy frequency and $c$ the adiabatic sound speed; and $\nabla \equiv\left(\partial_{x}, \partial_{y}\right)$. We consider a domain $\Omega=D \times\left[z_{1}, z_{2}\right]$; to allow maximum generality, the horizontal domain $D$ is assumed to be bounded by $J$ smooth simple closed curves $\partial D_{j}, j=1, \ldots, J$, where it is understood that the domain may be periodic in one direction (in which case some of the boundary elements will be closed after invoking periodicity). This includes the important case of a periodic zonal channel. The boundary conditions on $\partial D_{j}$ are those of no normal flow and conservation of circulation at each vertical level $z$ :

$$
\Phi_{s}=0
$$

and

$$
\frac{\partial}{\partial t} \oint_{\partial D_{j}} \Phi_{n} \mathrm{~d} s=0 \quad \text { on } \partial D_{j} \quad(j=1, \ldots, J),
$$

where the subscripts $s$ (arclength along $\partial D$ ) and $n$ (unit outward normal to $\partial D$ ) refer respectively to the tangential and normal derivatives on the curves $\partial D_{j}(j=1, \ldots, J)$. The boundary conditions on the lower and upper horizontal surfaces $z=z_{1}, z_{2}$ are

$$
\frac{\mathrm{D} \Lambda_{i}}{\mathrm{D} t} \equiv \Lambda_{i t}+\partial\left(\Phi_{i}, \Lambda_{i}\right)=0 \quad \text { on } z=z_{i} \quad(i=1,2),
$$

where $A_{i} \equiv \Phi_{z i}-B_{i} \Phi_{i}+f S_{i} \eta_{i}, B \equiv N^{2} / g$, and $\eta_{i}$ is the topography, if any (normally $\eta_{2}=0$ ). Here and henceforth the subscript $i=1,2$ denotes the value on $z=z_{i}$. The 
standard quasi-geostrophic model (e.g. Pedlosky 1987) is recovered in the special case $B=0$ and $c=\infty$ (see White 1977). In Appendix A, the property

$$
\frac{\mathrm{d}}{\mathrm{d} t} \iint_{D} \Phi \mathrm{d} x \mathrm{~d} y=0 \quad \forall z
$$

is established; for $B \neq 0$ this condition can be derived, while for $B=0$ it is simply a constraint that can be imposed without loss of generality.

The above model conserves an energy (White 1977), given by

$$
\begin{aligned}
\mathscr{E}[\Phi] & =\iiint_{\Omega} \frac{\rho}{2}\left\{|\nabla \Phi|^{2}+\frac{1}{S} \Phi_{z}^{2}\right\} \mathrm{d} x \mathrm{~d} y \mathrm{~d} z-\left.\iint_{D} \frac{r_{i} B_{i}}{2} \Phi_{i}^{2} \mathrm{~d} x \mathrm{~d} y\right|_{i=1} ^{i=2} \\
& =\iiint_{\Omega} \frac{\rho}{2}\left\{|\nabla \Phi|^{2}+\frac{1}{S}\left(\Phi_{z}-B \Phi\right)^{2}+\frac{f^{2}}{c^{2}} \Phi^{2}\right\} \mathrm{d} x \mathrm{~d} y \mathrm{~d} z
\end{aligned}
$$

The material conservation of $P$ in the interior (2.1), and of $A_{i}$ on the horizontal boundaries (2.4), together with conservation of circulation along the vertical boundaries $(2.3 b)$, implies the conservation of Casimir invariants of the form (Shepherd 1989)

$\mathscr{C}[\Phi]=\iiint_{\Omega} \rho G_{3}(P ; z) \mathrm{d} x \mathrm{~d} y \mathrm{~d} z-\left.\iint_{D} r_{i} G_{i}\left(A_{i}\right) \mathrm{d} x \mathrm{~d} y\right|_{i=1} ^{i=2}-\int_{z_{1}}^{z_{2}}\left(\rho \sum_{j=1}^{J} \Gamma_{j}(z) \oint_{\partial D_{j}} \Phi_{n} \mathrm{~d} s\right) \mathrm{d} z ;$

here $G_{3}(\xi ; z)$ is an arbitrary function of $\xi$ for each $z ; G_{i}(\xi)$ are arbitrary functions of $\xi$ for $i=1,2$; and $\Gamma_{j}(z)$ are arbitrary functions of $z$ for $j=1, \ldots, J$. When the dynamics are zonally symmetric (i.e. invariant under translations in $x$ ), which requires that $\eta_{i}=\eta_{i}(y)$ and that the lateral boundaries $\partial D_{j}$ be independent of $x$, then the model also conserves a zonal impulse (Shepherd 1989):

$$
\mathscr{M}[\Phi]=\iiint_{\Omega} \rho y P \mathrm{~d} x \mathrm{~d} y \mathrm{~d} z-\left.\iint_{D} r_{i} y A_{i} \mathrm{~d} x \mathrm{~d} y\right|_{i=1} ^{i=2}
$$

\section{The disturbance problem}

We will consider the stability of a steady basic state $\Phi=\Psi, P=Q, \Lambda_{i}=\Theta_{i}$, satisfying functional relations $\Psi=\Psi(Q)$ for each $z$ and $\Psi_{i}=\Psi_{i}\left(\Theta_{i}\right)$ for $i=1,2$. (The special cases of spatially uniform $Q$ or $\Theta_{i}$, for which some of these functions fail to exist, are treated separately in $\$ 6$ and $\$ 7.1$ respectively.) In general these basic states may be non-parallel. If the problem is zonally symmetric, however, then we restrict ourselves to zonally symmetric basic states - it will be shown in the Discussion, following the arguments of Andrews (1984), that only such basic states can satisfy our stability criteria - in which case $Q=Q(y, z)$ and $\Theta_{i}=\Theta_{i}(y)$. Therefore for any such basic state we may introduce the functions $\Psi_{3}^{\alpha}(\xi ; z)$ and $\Psi_{i}^{\alpha}(\xi)(i=1,2)$ defined by

$$
\begin{gathered}
\Psi_{3}^{\alpha}(Q ; z)=\Psi+\alpha y, \\
\Psi_{i}^{\alpha}\left(\Theta_{i}\right)=\Psi_{i}+\alpha y \quad(i=1,2) .
\end{gathered}
$$

In the case of zonally symmetric basic states, $\alpha$ is a free parameter; otherwise $\alpha=0$. Now, if there exists an allowable value of $\alpha$ for which $\mathrm{d} \Psi_{3}^{\alpha} / \mathrm{d} Q>0, \mathrm{~d} \Psi_{1}^{\alpha} / \mathrm{d} \Theta_{1}>0$, and $\mathrm{d} \Psi_{2}^{\alpha} / \mathrm{d} \Theta_{2}<0$ over the domains of definition of these functions, then the basic flow is provably stable by the quasi-geostrophic form of Arnol'd's first stability theorem 
(McIntyre \& Shepherd 1987, Appendix B; Kushner \& Shepherd 1995, Appendix $\mathrm{B})$. We are interested here in the case where these inequalities are reversed, which corresponds to Arnol'd's second stability theorem. We therefore consider basic states satisfying

$$
\begin{gathered}
-\frac{\mathrm{d} \Psi_{3}^{\alpha}}{\mathrm{d} Q} \geqslant C_{3}>0, \\
(-1)^{i} \frac{\mathrm{d} \Psi_{i}^{\alpha}}{\mathrm{d} \Theta_{i}} \geqslant C_{i}>0 \quad(i=1,2),
\end{gathered}
$$

and determine conditions under which they may be proved to be nonlinearly stable. It is worth emphasizing that the meteorologically important case of a zonal flow with eastward (positive) vertical shear, $Q_{y}>0$, and $\Theta_{i y}<0$, falls within this scenario; such a flow cannot be made to satisfy the conditions of Arnol'd's first theorem (cf. Andrews 1984).

Note that if the conditions (3.2) hold, then the functions $\Psi_{3}^{\alpha}(\xi ; z)$ and $\Psi_{i}^{\alpha}(\xi)$ $(i=1,2)$ may always be extended outside the ranges of definition determined by (3.1) in such a way that the inequalities (3.2) continue to hold (cf. Arnol'd 1966).

Now consider the finite-amplitude disturbance problem

$$
P=Q+q, \quad \Phi=\Psi+\psi, \quad A_{i}=\Theta_{i}+\theta_{i} \quad(i=1,2),
$$

for which $q=\nabla^{2} \psi+(1 / \rho)\left(r \psi_{z}\right)_{z}$ and $\theta_{i}=\psi_{z i}-B_{i} \psi_{i}$. By nonlinear stability of a basic state we mean that certain disturbance norms can be shown to be bounded for all time by functionals of the initial disturbance that go to zero as the initial disturbance fields go to zero. The disturbance norms of interest in this case are the (square roots of the) following functionals: the disturbance energy $\mathscr{E}[\psi]$ (where $\mathscr{E}[\cdot]$ is defined by (2.6)), the disturbance potential enstrophy

$$
\mathscr{Z}[\psi]=\iiint_{\Omega} \frac{\rho}{2} q^{2} \mathrm{~d} x \mathrm{~d} y \mathrm{~d} z
$$

and the disturbance available potential energy on the horizontal boundaries

$$
\mathscr{P}_{i}[\psi]=\iint_{D} \frac{r_{i}}{2} \theta_{i}^{2} \mathrm{~d} x \mathrm{~d} y .
$$

In this regard we will use the disturbance pseudoenergy/momentum

$$
\mathscr{A} \equiv(\mathscr{E}-\alpha \mathscr{M}+\mathscr{C})[\Phi]-(\mathscr{E}-\alpha \mathscr{M}+\mathscr{C})[\Psi]
$$

which is evidently an exact invariant of the nonlinear dynamics: $\mathrm{d} \mathscr{A} / \mathrm{d} t=0$. If we choose

$$
G_{3}(\xi ; z)=\int^{\xi} \Psi_{3}^{\alpha}(\xi ; z) \mathrm{d} \xi, \quad G_{i}(\xi)=\int^{\xi} \Psi_{i}^{\alpha}(\xi) \mathrm{d} \xi \quad(i=1,2), \quad \Gamma_{j}(z)=\left.\Psi\right|_{\partial D_{j}}
$$

in (2.7), then we have $\delta \mathscr{A}=0$ and $\mathscr{A}$ may be shown to take the form

$$
\begin{aligned}
\mathscr{A}=\mathscr{E}[\psi] & +\iiint_{\Omega}\left\{\rho \int_{Q}^{Q+q}\left[\Psi_{3}^{\alpha}(\xi ; z)-\Psi_{3}^{\alpha}(Q ; z)\right] \mathrm{d} \xi\right\} \mathrm{d} x \mathrm{~d} y \mathrm{~d} z \\
& -\left.\iint_{D}\left\{r_{i} \int_{\Theta_{i}}^{\Theta_{i}+\theta_{i}}\left[\Psi_{i}^{\alpha}(\xi)-\Psi_{i}^{\alpha}\left(\Theta_{i}\right)\right] \mathrm{d} \xi\right\} \mathrm{d} x \mathrm{~d} y\right|_{i=1} ^{i=2} .
\end{aligned}
$$




\section{Inequalities}

In the case of Arnol'd's first stability theorem, $\mathscr{A}$ is positive definite and this fact may be used to prove nonlinear stability in a straightforward manner (see e.g. McIntyre \& Shepherd 1987, Appendix B). But in the present case, we see from (3.2) that the explicit integrands in (3.8) are negative definite:

$$
\begin{aligned}
& \iiint_{\Omega}\left\{\rho \int_{Q}^{Q+q}\left[\Psi_{3}^{\alpha}(\xi ; z)-\Psi_{3}^{\alpha}(Q ; z)\right] \mathrm{d} \xi\right\} \mathrm{d} x \mathrm{~d} y \mathrm{~d} z \leqslant-C_{3} \mathscr{Z}[\psi], \\
& (-1)^{i+1} \iint_{D}\left\{r_{i} \int_{\Theta_{i}}^{\Theta_{i}+\theta_{i}}\left[\Psi_{i}^{\alpha}(\xi)-\Psi_{i}^{\alpha}\left(\Theta_{i}\right)\right] \mathrm{d} \xi\right\} \mathrm{d} x \mathrm{~d} y \leqslant-C_{i} \mathscr{P}_{i}[\psi] .
\end{aligned}
$$

Since $\mathscr{E}[\psi]>0$, this means that $\mathscr{A}$ is, in principle, of indefinite sign. For homogeneous boundary conditions (i.e. with zero disturbance circulation and with $\theta_{i}=0$ ), it is possible to show that under certain circumstances $\mathscr{A}$ is negative definite, in which case nonlinear stability may be proved (McIntyre \& Shepherd 1987; see also $§ 7.1$ of the present paper) - this is the straightforward version of Arnol'd's second theorem, which involves the use of a Poincare inequality to bound $\mathscr{E}[\psi]$ in terms of $\mathscr{Z}[\psi]$. However we wish to consider general boundary conditions, for which $\mathscr{E}[\psi]$ cannot be so bounded.

To overcome this difficulty, we divide the disturbance fields into two components as follows:

$$
q=q_{0}+q^{\prime}, \quad \psi=\psi_{0}+\psi^{\prime}, \quad \theta_{i}=\theta_{i 0}+\theta_{i}^{\prime} \quad(i=1,2),
$$

where subscript zero quantities are the initial values, at $t=0$. The primed quantities may be seen to satisfy

$$
\begin{gathered}
\psi_{s}^{\prime}=0 \quad \text { and } \quad \oint_{\partial D_{j}} \psi_{n}^{\prime} \mathrm{d} s=0 \quad \text { on } \partial D_{j} \quad(j=1, \ldots, J), \\
\iint_{D} q^{\prime} \mathrm{d} x \mathrm{~d} y=0=\iint_{D} \psi^{\prime} \mathrm{d} x \mathrm{~d} y \quad \forall z
\end{gathered}
$$

It can be verified that $\mathscr{E}\left[\psi^{\prime}\right]$ satisfies

$$
\mathscr{E}\left[\psi^{\prime}\right]=-\iiint_{\Omega} \frac{\rho}{2} \psi^{\prime} q^{\prime} \mathrm{d} x \mathrm{~d} y \mathrm{~d} z+\left.\iint_{D} \frac{r_{i}}{2} \psi_{i}^{\prime} \theta_{i}^{\prime} \mathrm{d} x \mathrm{~d} y\right|_{i=1} ^{i=2} .
$$

We will now show that $\mathscr{E}\left[\psi^{\prime}\right]$ can be bounded in terms of $\mathscr{L}\left[\psi^{\prime}\right]$ and $\mathscr{P}_{i}\left[\psi^{\prime}\right]$ through a suitable Poincaré inequality.

We first establish a bound on the horizontal derivatives in $\mathscr{E}\left[\psi^{\prime}\right]$. Let

$$
\lambda=\min \frac{\iint_{D}|\nabla u|^{2} \mathrm{~d} x \mathrm{~d} y}{\iint_{D} u^{2} \mathrm{~d} x \mathrm{~d} y}
$$

where $u$ ranges over all non-zero differentiable functions of $x, y$ subject to the conditions

$$
u_{s}=0 \quad \text { on } \partial D_{j} \quad(j=1, \ldots, J), \quad \iint_{D} u \mathrm{~d} x \mathrm{~d} y=0 .
$$

It may be verified that $\lambda$ is the lowest non-trivial eigenvalue of the problem

$$
\nabla^{2} u+\lambda u=0 \quad \text { in } D
$$




$$
u_{s}=0 \quad \text { and } \quad \oint_{\partial D_{j}} u_{n} \mathrm{~d} s=0 \quad \text { on } \partial D_{j} \quad(j=1, \ldots, J)
$$

Since $\psi^{\prime}$ satisfies $(4.8)$, we obtain the inequality

$$
\iiint_{\Omega} \frac{\rho}{2}\left|\nabla \psi^{\prime}\right|^{2} \mathrm{~d} x \mathrm{~d} y \mathrm{~d} z \geqslant \lambda \iiint_{\Omega} \frac{\rho}{2}\left(\psi^{\prime}\right)^{2} \mathrm{~d} x \mathrm{~d} y \mathrm{~d} z
$$

We next establish a bound on the vertical derivatives in $\mathscr{E}\left[\psi^{\prime}\right]$. Let

$$
\mu_{0}(K) \equiv \min \frac{\int_{z_{1}}^{z_{2}} r v_{z}^{2} \mathrm{~d} z+\sum_{i=1}^{2}\left[(-1)^{i+1} B_{i}-\frac{K}{C_{i}}\right] r_{i} v^{2}\left(z_{i}\right)}{\int_{z_{1}}^{z_{2}} \rho v^{2} \mathrm{~d} z},
$$

where $K=$ const. $\geqslant 0$ and $v$ ranges over all non-zero differentiable functions of $z$. It may be verified that $\mu_{0}(K)$ is the lowest eigenvalue of the problem

$$
\begin{gathered}
\left(r v_{z}\right)_{z}+\mu \rho v=0 \quad \forall z \\
C_{i} v_{z}\left(z_{i}\right)=\left[C_{i} B_{i}+(-1)^{i} K\right] v\left(z_{i}\right) \quad(i=1,2) .
\end{gathered}
$$

Letting $v=\psi^{\prime}$, (4.12) implies

$$
\int_{z_{1}}^{z_{2}} \frac{r}{2}\left(\psi_{z}^{\prime}\right)^{2} \mathrm{~d} z-\left.\frac{r_{i} B_{i}}{2}\left(\psi_{i}^{\prime}\right)^{2}\right|_{i=1} ^{i=2} \geqslant \mu_{0}(K) \int_{z_{1}}^{z_{2}} \frac{\rho}{2}\left(\psi^{\prime}\right)^{2} \mathrm{~d} z+\sum_{i=1}^{2} \frac{K r_{i}}{2 C_{i}}\left(\psi_{i}^{\prime}\right)^{2} .
$$

Combining (4.11) and (4.15) yields

$$
\mathscr{E}\left[\psi^{\prime}\right] \geqslant\left[\lambda+\mu_{0}(K)\right] \iiint_{\Omega} \frac{\rho}{2}\left(\psi^{\prime}\right)^{2} \mathrm{~d} x \mathrm{~d} y \mathrm{~d} z+\sum_{i=1}^{2} \frac{K}{C_{i}} \iint_{D} \frac{r_{i}}{2}\left(\psi_{i}^{\prime}\right)^{2} \mathrm{~d} x \mathrm{~d} y \equiv \mathscr{G}
$$

We now fix the value of $K$ by imposing the condition

$$
\lambda+\mu_{0}(K)=\frac{K}{C_{3}} .
$$

To see that (4.17) has a unique positive solution, note that $\mu_{0}(K)$ is a monotonically decreasing function of $K$, and that $\mu_{0}(0) \geqslant 0$. (For the standard quasi-geostrophic model, $\mu_{0}(0)=0$; for the modified model, it can be shown - cf. $(2.6 a, b)-$ that $\mu_{0}(0)>0$.) Therefore between $K=0$ and $K=\infty$, the left-hand side of (4.17) is decreasing from $\lambda+\mu_{0}(0)>0$ to $-\infty$ while the right-hand side of (4.17) is increasing from 0 to $\infty$; it follows that $(4.17)$ has a unique positive solution. Using (4.6) and applying the Hölder inequality yields

$$
\mathscr{E}\left[\psi^{\prime}\right] \leqslant \mathscr{G}^{1 / 2}\left(\begin{array}{c}
C_{3} \mathscr{Z}\left[\psi^{\prime}\right]+\sum_{i=1}^{2} C_{i} \mathscr{P}_{i}\left[\psi^{\prime}\right] \\
-\frac{1}{K}
\end{array}\right)^{1 / 2}
$$

Finally, combining (4.16) and (4.18), and using (4.17), yields the desired Poincare inequality

$$
\mathscr{E}\left[\psi^{\prime}\right] \leqslant \frac{C_{3} \mathscr{Z}\left[\psi^{\prime}\right]+\sum_{i=1}^{2} C_{i} \mathscr{P}_{i}\left[\psi^{\prime}\right]}{K}
$$


That this Poincare inequality is in fact the best possible is easily seen by substituting $\psi^{\prime}=u^{1} v^{0}$ in (4.19), where $u^{1}$ is the first non-trivial eigenfunction of (4.9)-(4.10) and $v^{0}$ is the first eigenfunction of (4.13)-(4.14), in which case (4.19) becomes an equality.

We now need to express (4.19) in terms of initial and total disturbance fields. It is convenient to make an additional decomposition, namely

$$
q=\bar{q}+\tilde{q}, \quad \psi=\bar{\psi}+\tilde{\psi}, \quad \theta_{i}=\bar{\theta}_{i}+\tilde{\theta}_{i} \quad(i=1,2)
$$

where the overbar denotes the horizontal average over $D$. Since the overbar fields are time independent, it may be seen that

$$
q^{\prime}=\tilde{q}-\tilde{q}_{0}, \quad \psi^{\prime}=\tilde{\psi}-\tilde{\psi}_{0}, \quad \theta_{i}^{\prime}=\tilde{\theta}_{i}-\tilde{\theta}_{i 0} \quad(i=1,2) .
$$

Substituting (4.21) into (4.19) produces

$$
\mathscr{E}\left[\psi^{\prime}\right] \leqslant \frac{C_{3} \mathscr{Z}[\tilde{\psi}]+\sum_{i=1}^{2} C_{i} \mathscr{P}_{i}[\tilde{\psi}]}{K}+\frac{C_{3} \mathscr{Z}\left[\tilde{\psi}_{0}\right]+\sum_{i=1}^{2} C_{i} \mathscr{P}_{i}\left[\tilde{\psi}_{0}\right]}{K}
$$

As for $\mathscr{E}\left[\psi^{\prime}\right]$ itself, it may be verified from (4.6) that the following identity holds:

$$
\mathscr{E}\left[\tilde{\psi}_{0}\right]-\mathscr{E}[\tilde{\psi}]=-\mathscr{E}\left[\psi^{\prime}\right]+\iiint_{\Omega} \rho \tilde{\psi}_{0}\left(\tilde{q}-\tilde{q}_{0}\right) \mathrm{d} x \mathrm{~d} y \mathrm{~d} z+\left.\iint_{D} r_{i} \tilde{\psi}_{i 0}\left(\tilde{\theta}_{i 0}-\tilde{\theta}_{i}\right) \mathrm{d} x \mathrm{~d} y\right|_{i=1} ^{i=2}
$$

(This rather curious combination of terms is used in order to eliminate the circulation terms.) Then combining (4.22) with (4.23) and using the Hölder inequality yields the bound

$$
\mathscr{E}[\tilde{\psi}] \leqslant \frac{\mathscr{W}^{2}+2 \mathscr{N} \mathscr{W}+\mathscr{J}}{K}+\mathscr{E}\left[\tilde{\psi}_{0}\right]
$$

where

$$
\mathscr{W} \equiv\left\{C_{3} \mathscr{Z}[\tilde{\psi}]+\sum_{i=1}^{2} C_{i} \mathscr{P}_{i}[\tilde{\psi}]\right\}^{1 / 2}
$$

$\mathscr{N} \equiv\left\{\iiint_{\Omega} \frac{\rho C_{3}}{2}\left(\tilde{q}_{0}+\frac{K}{C_{3}} \tilde{\psi}_{0}\right)^{2} \mathrm{~d} x \mathrm{~d} y \mathrm{~d} z+\sum_{i=1}^{2} \iint_{D} \frac{r_{i} C_{i}}{2}\left(\tilde{\theta}_{i 0}-(-1)^{i} \frac{K}{C_{i}} \tilde{\psi}_{i 0}\right)^{2} \mathrm{~d} x \mathrm{~d} y\right\}^{1 / 2}$,

$\mathscr{J} \equiv C_{3} \mathscr{Z}\left[\tilde{\psi}_{0}\right]+\sum_{i=1}^{2} C_{i} \mathscr{P}_{i}\left[\tilde{\psi}_{0}\right]+K\left\{\iiint_{\Omega} \rho \tilde{q}_{0} \tilde{\psi}_{0} \mathrm{~d} x \mathrm{~d} y \mathrm{~d} z-\sum_{i=1}^{2}(-1)^{i} \iint_{D} r_{i} \tilde{\theta}_{i 0} \tilde{\psi}_{i 0} \mathrm{~d} x \mathrm{~d} y\right\}$.

Note that $\mathcal{N}, \mathscr{J}$ and $\mathscr{E}\left[\tilde{\psi}_{0}\right]$ are all functionals of the initial disturbance that go to zero as the initial disturbance goes to zero. All that remains, therefore, is to bound $\mathscr{W}$.

Returning to the disturbance pseudoenergy/momentum (3.8), using (4.1) and (4.2) together with the fact that

$$
\mathscr{Z}[\psi]=\mathscr{Z}\left[\bar{\psi}_{0}\right]+\mathscr{Z}[\tilde{\psi}], \quad \mathscr{P}_{i}[\psi]=\mathscr{P}_{i}\left[\bar{\psi}_{0}\right]+\mathscr{P}_{i}[\tilde{\psi}],
$$

yields the inequality

$$
\mathscr{W}^{2} \leqslant \mathscr{E}[\psi]-\mathscr{A}-\overline{\mathscr{W}}_{0}^{2}
$$


where

$$
\overline{\mathscr{W}}_{0}^{2} \equiv C_{3} \mathscr{Z}\left[\bar{\psi}_{0}\right]+\sum_{i=1}^{2} C_{i} \mathscr{P}_{i}\left[\bar{\psi}_{0}\right]
$$

Combining (4.24) with (4.27) then gives

$$
(K-1) \mathscr{W}^{2}-2 \mathscr{N} \mathscr{W} \leqslant K\left(\mathscr{E}\left[\psi_{0}\right]-\mathscr{A}-\overline{\mathscr{W}}_{0}^{2}\right)+\mathscr{J},
$$

after noting that

$$
\mathscr{E}[\psi]=\mathscr{E}\left[\bar{\psi}_{0}\right]+\mathscr{E}[\tilde{\psi}], \quad \mathscr{E}\left[\psi_{0}\right]=\mathscr{E}\left[\bar{\psi}_{0}\right]+\mathscr{E}\left[\tilde{\psi}_{0}\right]
$$

Now suppose that

$$
K=1+k, \quad k>0 .
$$

Whenever (4.31) holds, it follows from (4.29) that

$$
\mathscr{W} \leqslant \frac{\mathscr{N}+\left\{\mathscr{N}^{2}+k\left[K\left(\mathscr{E}\left[\psi_{0}\right]-\mathscr{A}-\overline{\mathscr{W}}_{0}^{2}\right)+\mathscr{J}\right]\right\}^{1 / 2}}{k} .
$$

This is all we need to prove stability: the right-hand side of (4.32) is a functional of the initial disturbance that goes to zero as the initial disturbance goes to zero, and this upper bound on $\mathscr{W}$ may be used to bound the disturbance norms of interest as will be seen in the next section. The crucial question concerns determining when $(4.31)$ is true.

\section{Nonlinear stability theorem}

Whenever (4.31) is true, it follows from (4.25a), (4.26) and (4.32) that the disturbance quantities $\mathscr{Z}[\psi]$ and $\mathscr{P}_{i}[\psi]$ can be bounded for all time according to

$$
\begin{aligned}
\mathscr{Z}[\psi] & \leqslant \mathscr{Z}\left[\bar{\psi}_{0}\right]+\frac{\mathscr{W}^{2}}{C_{3}} \\
& \leqslant \mathscr{Z}\left[\bar{\psi}_{0}\right]+\frac{\left(\mathscr{N}+\left\{\mathscr{N}^{2}+k\left[K\left(\mathscr{E}\left[\psi_{0}\right]-\mathscr{A}-\overline{\mathscr{W}}_{0}^{2}\right)+\mathscr{J}\right]\right\}^{1 / 2}\right)^{2}}{C_{3} k^{2}} \\
\mathscr{P}_{i}[\psi] & \leqslant \mathscr{P}_{i}\left[\bar{\psi}_{0}\right]+\frac{\mathscr{W}^{2}}{C_{i}} \\
& \leqslant \mathscr{P}_{i}\left[\bar{\psi}_{0}\right]+\frac{\left(\mathscr{N}+\left\{\mathscr{N}^{2}+k\left[K\left(\mathscr{E}\left[\psi_{0}\right]-\mathscr{A}-\overline{\mathscr{W}}_{0}^{2}\right)+\mathscr{J}\right]\right\}^{1 / 2}\right)^{2}}{C_{i} k^{2}}(i=1,2)
\end{aligned}
$$

Furthermore, by (4.24), (4.30) and (4.32) we have

$$
\mathscr{E}[\psi] \leqslant \frac{\left(\mathscr{N}+\left\{\mathscr{N}^{2}+k\left[K\left(\mathscr{E}\left[\psi_{0}\right]-\mathscr{A}-\overline{\mathscr{W}}_{0}^{2}\right)+\mathscr{J}\right]\right\}^{1 / 2}\right)^{2}}{k^{2}}+\mathscr{A}+\overline{\mathscr{W}}_{0}^{2}
$$

These expressions provide the desired bounds on the disturbance norms of interest in terms of the initial disturbance fields: in all cases the bounds go to zero as the initial disturbance goes to zero. 
Liapunov stability can be established whenever $-\mathrm{d} \Psi_{3}^{\alpha} / \mathrm{d} Q$ and $(-1)^{i} \mathrm{~d} \Psi_{i}^{\alpha} / \mathrm{d} \Theta_{i}$ are bounded away from infinity, namely

$$
\begin{gathered}
-\frac{\mathrm{d} \Psi_{3}^{\alpha}}{\mathrm{d} Q} \leqslant \bar{C}_{3}<\infty, \\
(-1)^{i} \frac{\mathrm{d} \Psi_{i}^{\alpha}}{\mathrm{d} \Theta_{i}} \leqslant \bar{C}_{i}<\infty \quad(i=1,2),
\end{gathered}
$$

in addition to $(3.2 a, b)$. We consider the disturbance norm defined by

$$
\|\psi\| \equiv\left\{C_{3} \mathscr{Z}[\psi]+\sum_{i=1}^{2} C_{i} \mathscr{P}_{i}[\psi]\right\}^{1 / 2}+\{K \mathscr{E}[\psi]\}^{1 / 2} .
$$

The first task is to bound $\|\psi\|$ from above. Using (4.26) and (4.28), we obtain

$$
\|\psi\|=\left\{\overline{\mathscr{W}}_{0}^{2}+\mathscr{W}^{2}\right\}^{1 / 2}+\{K \mathscr{E}[\psi]\}^{1 / 2},
$$

and $\mathscr{W}^{2}$ and $\mathscr{E}[\psi]$ can be bounded in terms of initial quantities by (4.32) and (5.3). The next task is to bound $\left\|\psi_{0}\right\|$ from below. First note that since $\tilde{\psi}_{0}$ satisfies (4.7), we have (cf. (4.16))

$$
\frac{1}{C_{3}} \iiint_{\Omega} \frac{\rho}{2}\left(\tilde{\psi}_{0}\right)^{2} \mathrm{~d} x \mathrm{~d} y \mathrm{~d} z+\sum_{i=1}^{2} \frac{1}{C_{i}} \iint_{D} \frac{r_{i}}{2}\left(\tilde{\psi}_{i 0}\right)^{2} \mathrm{~d} x \mathrm{~d} y \leqslant \frac{\mathscr{E}\left[\tilde{\psi}_{0}\right]}{K} .
$$

Using (5.7) together with the Hölder inequality then yields

$$
\mathscr{N} \leqslant \mathscr{W}_{0}+\left\{K \mathscr{E}\left[\tilde{\psi}_{0}\right]\right\}^{1 / 2}, \quad \mathscr{J} \leqslant \mathscr{W}_{0}^{2}+2 \mathscr{W}_{0}\left\{K \mathscr{E}\left[\tilde{\psi}_{0}\right]\right\}^{1 / 2}
$$

where

$$
\mathscr{W}_{0}^{2} \equiv C_{3} \mathscr{Z}\left[\tilde{\psi}_{0}\right]+\sum_{i=1}^{2} C_{i} \mathscr{P}_{i}\left[\tilde{\psi}_{0}\right]
$$

and hence

$$
\mathscr{N} \leqslant\left\|\psi_{0}\right\|, \quad \mathscr{J} \leqslant\left\|\psi_{0}\right\|^{2}
$$

Also, from (3.8) and (5.4),

$$
\mathscr{E}\left[\psi_{0}\right]-\mathscr{A} \leqslant \bar{C}_{3} \mathscr{Z}\left[\psi_{0}\right]+\sum_{i=1}^{2} \bar{C}_{i} \mathscr{P}_{i}\left[\psi_{0}\right] \leqslant \max _{i=1,2,3}\left\{\frac{\bar{C}_{i}}{C_{i}}\right\}\left\|\psi_{0}\right\|^{2} .
$$

Combining (5.10) and (5.11) with (4.32) then yields

$$
\mathscr{W} \leqslant \frac{1}{k}\left\{1+\left[k\left(1+K \max _{i}\left\{\bar{C}_{i} / C_{i}\right\}\right)+1\right]^{1 / 2}\right\}\left\|\psi_{0}\right\| .
$$

It remains to bound $\overline{\mathscr{W}}_{0}^{2}$ and $\mathscr{E}[\psi]$ from above by $\left\|\psi_{0}\right\|$. Evidently

$$
\overline{\mathscr{W}}_{0}^{2} \leqslant\left\|\bar{\psi}_{0}\right\|^{2} \leqslant\left\|\psi_{0}\right\|^{2},
$$

while from (4.24), (4.30), (5.10) and (5.12),

$$
K \mathscr{E}[\psi] \leqslant\left(\left[\frac{1}{k}\left\{1+\left[k\left(1+K \max _{i}\left\{\bar{C}_{i} / C_{i}\right\}\right)+1\right]^{1 / 2}\right\}+1\right]^{2}+1\right)\left\|\psi_{0}\right\|^{2} .
$$


Finally, combining (5.6) with (5.12)-(5.14) yields

$$
\|\psi\| \leqslant 2\left(\left[\frac{1}{k}\left\{1+\left[k\left(1+K \max _{i}\left\{\bar{C}_{i} / C_{i}\right\}\right)+1\right]^{1 / 2}\right\}+1\right]^{2}+1\right)^{1 / 2}\left\|\psi_{0}\right\| .
$$

The inequality (5.15) establishes nonlinear Liapunov stability in the norm (5.5), whenever (4.31) is true. Note that as $k \rightarrow 0$, the stability (maximum amplification) factor implied by (5.15) diverges, as expected.

We now derive a condition on the basic state such that (4.31) is true, where $K$ is determined by (4.17). This requires that an explicit expression for $\mu_{0}(K)$ be obtained in terms of basic-state quantities. To derive such an expression, consider the eigenvalue problem (4.13)-(4.14). The interior equation (4.13) is second order and therefore has two independent solutions: let $U(z, \mu)$ and $V(z, \mu)$ be the two solutions satisfying

$$
U\left(z_{1}, \mu\right)=1, \quad U_{z}\left(z_{1}, \mu\right)=0 ; \quad V\left(z_{1}, \mu\right)=0, \quad V_{z}\left(z_{1}, \mu\right)=1 .
$$

The linear combination of these two solutions (within a non-zero constant factor) that satisfies the boundary condition (4.14) at $z=z_{1}$ is given by

$$
v=C_{1} U(z, \mu)-\left[K-C_{1} B_{1}\right] V(z, \mu) .
$$

Imposing the boundary condition (4.14) at $z=z_{2}$ then yields

$$
C_{1}\left\{C_{2} U_{z}\left(z_{2}, \mu\right)-\left[K+C_{2} B_{2}\right] U\left(z_{2}, \mu\right)\right\}=\left[K-C_{1} B_{1}\right]\left\{C_{2} V_{z}\left(z_{2}, \mu\right)-\left[K+C_{2} B_{2}\right] V\left(z_{2}, \mu\right)\right\} \text {. }
$$

The eigenvalue problem (4.13)-(4.14) is thus equivalently represented by (5.18): for every $K,(5.18)$ determines an infinite number of eigenvalues $\mu_{0}(K), \mu_{1}(K), \ldots$ Conversely, we may regard (5.18) as an equation in $K$ for given $\mu$ : for $\mu<\mu_{0}(0)$, this equation must have at least one solution with $K>0$, namely $K=K_{0}$ such that $\mu_{0}\left(K_{0}\right)=\mu$. (This is because $\mu_{0}(K)$ decreases from $\mu_{0}(0)$ to $-\infty$ as $K$ goes from 0 to $\infty$, and thus must take the value $\mu$ for some $K_{0}>0$.) This solution will moreover correspond to the smallest value of $K$ (for the given $\mu$ ).

Let

$$
\mu_{1}=\min \frac{\int_{z_{1}}^{z_{2}} r v_{z}^{2} \mathrm{~d} z}{\int_{z_{1}}^{z_{2}} \rho v^{2} \mathrm{~d} z} \quad \text { subject to } v\left(z_{1}\right)=0=v\left(z_{2}\right) .
$$

If $V\left(z_{2}, \mu\right)=0$ for some $\mu<\mu_{1}$, then $\mu_{1}$ is not the minimum and we obtain a contradiction; it follows that $V\left(z_{2}, \mu\right) \neq 0$ for all $\mu<\mu_{1}$. But (4.13) and (5.16) imply that $V V_{z}>0$ for all $z>z_{1}$, and hence that $V_{z}>0$ for all $z>z_{1}$. From this one can conclude (again using (4.13) and (5.16)) that $V\left(z_{2}, \mu\right)>0$ for all $\mu<0$. Finally, by continuity of $V\left(z_{2}, \mu\right)$ in $\mu$, we conclude that $V\left(z_{2}, \mu\right)>0$ for all $\mu<\mu_{1}$.

Using this fact that $V\left(z_{2}, \mu\right)>0,(5.18)$ has only two solutions for $\mu<\mu_{1}$ :

$$
\begin{aligned}
K= & M_{ \pm}(\mu) \equiv \frac{C_{1} U\left(z_{2}, \mu\right)+C_{2} V_{z}\left(z_{2}, \mu\right)+\left[C_{1} B_{1}-C_{2} B_{2}\right] V\left(z_{2}, \mu\right)}{2 V\left(z_{2}, \mu\right)} \\
& \pm \frac{\left[\left\{C_{1} U\left(z_{2}, \mu\right)-C_{2} V_{z}\left(z_{2}, \mu\right)+\left[C_{1} B_{1}+C_{2} B_{2}\right] V\left(z_{2}, \mu\right)\right\}^{2}+4 C_{1} C_{2}\left(r_{1} / r_{2}\right)\right]^{1 / 2}}{2 V\left(z_{2}, \mu\right)},(5.20)
\end{aligned}
$$

where we have used the fact that

$$
U\left(z_{2}, \mu\right) V_{z}\left(z_{2}, \mu\right)-U_{z}\left(z_{2}, \mu\right) V\left(z_{2}, \mu\right)=\frac{r_{1}}{r_{2}}
$$


which follows from (4.13) and (5.16). However, taking $v(z)=V\left(z, \mu_{1}\right)$ in the righthand side of (4.12) with $K=0$ implies that $\mu_{0}(0) \leqslant \mu_{1}$. Thus $\mu_{0}(K)$ with $K>0$ must be one of the solutions represented by $(5.20)$. Since the function $\mu_{0}(K)$ corresponds to the smallest value of $K$ for a given $\mu$, we have that $\mu_{0}(K)$ is the inverse of $M_{-}(\mu)$ : namely $\mu_{0}\left(M_{-}(\mu)\right)=\mu$ for all $\mu<\mu_{0}(0)$, or equivalently

$$
K=M_{-}\left(\mu_{0}(K)\right)
$$

for $K>0$. Now we substitute (4.17) into (5.22), and ask whether the solution of the resulting equation

$$
K=M_{-}\left(\frac{K}{C_{3}}-\lambda\right)
$$

occurs for $K>1$ : if it does, then (4.31) is true. Since the left-hand side of (5.23) is an increasing function of $K$ while the right-hand side is a decreasing function of $K$, and they must cross for some $K$, it is clear that the solution occurs for $K>1$ if and only if the right-hand side of (5.23) is greater than unity when $K=1$. Thus (4.31) is true if and only if

$$
M_{-}(m)>1 \text { and } m<\mu_{0}(0) \text {, where } m \equiv \frac{1}{C_{3}}-\lambda .
$$

It may be seen from (5.20) that this condition is determinable solely in terms of basic-state quantities. Since $M_{-}(m)$ is a decreasing function of $m$, the condition (5.24) is equivalently stated as

$m<m_{0}, \quad$ where $\quad m \equiv \frac{1}{C_{3}}-\lambda \quad$ and $\quad M_{-}\left(m_{0}\right)=1 \quad$ with $\quad m_{0}<\mu_{0}(0) \quad$ defines $m_{0}$.

Note that in (5.24) and (5.25), $\mu_{0}(0)$ may be replaced by $\mu_{1}$, which may be easier to evaluate for the modified quasi-geostrophic model. (Recall that $\mu_{0}(0)=0$ for the standard model.)

This leads to the following nonlinear stability theorem:

THEOREM 5.1. A basic state $\left(\Psi, Q, \Theta_{i}\right)$ satisfying the properties (3.2) and (5.4) for some $\alpha$ is nonlinearly Liapunov stable in the norm (5.5) when (5.24) or equivalently (5.25) holds, where $\lambda$ and $\mu_{0}(0)$ are defined by (4.7) and (4.12) and $M_{-}(\cdot)$ is defined by (5.20). In (5.24) and (5.25), $\mu_{0}(0)$ may be replaced by $\mu_{1}$ as defined by (5.19). Under these conditions, the disturbance energy $\mathscr{E}[\psi]$, disturbance potential enstrophy $\mathscr{Z}[\psi]$, and disturbance available potential energy on the horizontal boundaries $\mathscr{P}_{i}[\psi]$, are bounded for all time in terms of the initial disturbance fields according to (5.1)-(5.3).

An explicit expression for $M_{-}(\mu)$ is provided in Appendix B for the special case of constant stratification and a constant density scale height.

\section{Basic states with uniform potential vorticity}

A separate treatment is required for basic states with horizontally uniform potential vorticity, for which the function $\Psi_{3}^{\alpha}(\xi ; z)$ does not exist. In this case, $(2.1)$ implies that the disturbance potential vorticity $q$ is materially conserved and the disturbance potential enstrophy is thus an exact invariant:

$$
\mathscr{Z}[\psi]=\mathscr{Z}\left[\psi_{0}\right] .
$$


(Note that the flow itself is not assumed to have uniform potential vorticity, only the basic state.) For a given basic state we may still define the functions $\Psi_{i}^{\alpha}(\xi)$ for $i=1,2$ according to $(3.1 b)$, and we consider basic states satisfying $(3.2 b)$ and $(5.4 b)$. Taking (3.7) except with $G_{3}(\xi ; z)=0$, the disturbance pseudoenergy/momentum (3.6) becomes

$\mathscr{A}=\mathscr{E}[\psi]-\iiint_{\Omega} \rho(\Psi+\alpha y) q \mathrm{~d} x \mathrm{~d} y \mathrm{~d} z-\left.\iint_{D}\left\{r_{i} \int_{\Theta_{i}}^{\Theta_{i}+\theta_{i}}\left[\Psi_{i}^{\alpha}(\xi)-\Psi_{i}^{\alpha}\left(\Theta_{i}\right)\right] \mathrm{d} \xi\right\} \mathrm{d} x \mathrm{~d} y\right|_{i=1} ^{i=2}$,

in place of (3.8). Note that $\mathscr{A}$ is now no longer quadratic in the disturbance, except in the special case $q=0$ corresponding to constant-potential-vorticity flows. Equations (4.3)-(4.16) follow as before, but instead of (4.17) we let $K$ satisfy

$$
\lambda+\mu_{0}(K)=a,
$$

where $a$ is an arbitrary constant satisfying $0<a<\lambda+\mu_{0}(0)$. (Note that (6.3) has a positive solution for $K$ for all $a$ in this range.) The inequalities (4.18)-(4.22) then apply with $C_{3}$ replaced by $K / a$, while (4.23) continues to hold. Using (6.1), one obtains (4.24) with the definitions $(4.25)$ replaced by

$$
\begin{gathered}
\mathscr{W} \equiv\left\{\sum_{i=1}^{2} C_{i} \mathscr{P}_{i}[\tilde{\psi}]\right\}^{1 / 2} \\
\mathscr{N} \equiv\left\{\sum_{i=1}^{2} \iint_{D} \frac{r_{i} C_{i}}{2}\left(\tilde{\theta}_{i 0}-(-1)^{i} \frac{K}{C_{i}} \tilde{\psi}_{i 0}\right)^{2} \mathrm{~d} x \mathrm{~d} y\right\}^{1 / 2}, \\
\mathscr{J} \equiv \frac{4 K}{a}\left(\mathscr{Z}\left[\tilde{\psi}_{0}\right]\right)^{1 / 2}\left\{\iiint_{\Omega} \frac{\rho}{2}\left(\tilde{q}_{0}+a \tilde{\psi}_{0}\right)^{2} \mathrm{~d} x \mathrm{~d} y \mathrm{~d} z\right\}^{1 / 2} \\
+\sum_{i=1}^{2} \iint_{D} \frac{r_{i} \tilde{\theta}_{i 0}}{2}\left(C_{i} \tilde{\theta}_{i 0}-2 K(-1)^{i} \tilde{\psi}_{i 0}\right) \mathrm{d} x \mathrm{~d} y .
\end{gathered}
$$

Now, using (4.2) on (6.2), together with the definition $(6.4 a)$, yields the inequality

$$
\mathscr{W}^{2} \leqslant \mathscr{E}[\psi]-\mathscr{A}+\mathscr{Y}-\overline{\mathscr{W}}_{0}^{2}
$$

in place of (4.27), where

$$
\overline{\mathscr{W}}_{0}^{2} \equiv \sum_{i=1}^{2} C_{i} \mathscr{P}_{i}\left[\bar{\psi}_{0}\right], \quad \mathscr{Y} \equiv 2\left(\mathscr{Z}\left[\psi_{0}\right]\right)^{1 / 2}\left\{\iiint_{\Omega} \frac{\rho}{2}(\Psi+\alpha y)^{2} \mathrm{~d} x \mathrm{~d} y \mathrm{~d} z\right\}^{1 / 2}
$$

Combining (4.24) with (6.5), and supposing that (4.31) holds, then gives

$$
\mathscr{W} \leqslant \frac{\mathscr{N}+\left\{\mathscr{N}^{2}+k\left[K\left(\mathscr{E}\left[\psi_{0}\right]-\mathscr{A}+\mathscr{Y}-\overline{\mathscr{W}}_{0}^{2}\right)+\mathscr{J}\right]\right\}^{1 / 2}}{k}
$$

in place of (4.32).

Whenever $K$ defined by (6.3) satisfies (4.31), it follows from (6.4a) and (6.7) that the disturbance quantity $\mathscr{P}_{i}[\psi]$ can be bounded for all time according to

$$
\begin{aligned}
\mathscr{P}_{i}[\psi] & \leqslant \mathscr{P}_{i}\left[\bar{\psi}_{0}\right]+\frac{\mathscr{W}^{2}}{C_{i}} \\
& \leqslant \mathscr{P}_{i}\left[\bar{\psi}_{0}\right]+\frac{\left(\mathscr{N}+\left\{\mathscr{N}^{2}+k\left[K\left(\mathscr{E}\left[\psi_{0}\right]-\mathscr{A}+\mathscr{Y}-\overline{\mathscr{W}}_{0}^{2}\right)+\mathscr{J}\right]\right\}^{1 / 2}\right)^{2}}{C_{i} k^{2}}(i=1,2)
\end{aligned}
$$


$\mathscr{Z}[\psi]$ is, of course, bounded trivially by (6.1). As for $\mathscr{E}[\psi]$, from (4.24) and (6.7) we have

$$
\mathscr{E}[\psi] \leqslant \frac{\left(\mathscr{N}+\left\{\mathscr{N}^{2}+k\left[K\left(\mathscr{E}\left[\psi_{0}\right]-\mathscr{A}+\mathscr{Y}-\overline{\mathscr{W}}_{0}^{2}\right)+\mathscr{J}\right]\right\}^{1 / 2}\right)^{2}}{k^{2}}+\mathscr{A}-\mathscr{Y}+\overline{\mathscr{W}}_{0}^{2}
$$

As before, these expressions provide the desired bounds on the disturbance norms of interest in terms of the initial disturbance fields: in all cases the bounds go to zero as the initial disturbance goes to zero. Liapunov stability follows as in $\S 5$.

It remains to determine a condition on the basic state such that $(4.31)$ is true. Equations (5.16)-(5.22) hold as before, but now we use (6.3) to substitute for $\mu_{0}(K)$ in (5.22), obtaining

$$
K=M_{-}(a-\lambda)
$$

in place of (5.23). The question is whether $K$ defined by (6.10) satisfies $K>1$ for some $a>0$. Since the right-hand side of (6.10) is a decreasing function of $a$, this is evidently the case if and only if

$$
M_{-}(-\lambda)>1
$$

or equivalently,

$$
\lambda>-m_{0}, \quad \text { where } M_{-}\left(m_{0}\right)=1 \text { with } m_{0}<\mu_{0}(0) \text { defines } m_{0} .
$$

This leads to the following nonlinear stability theorem:

THEOREM 6.1. A basic state $\left(\Psi, Q, \Theta_{i}\right)$ with horizontally uniform potential vorticity $Q$, and satisfying the properties (3.2b) and (5.4b) for some $\alpha$, is nonlinearly Liapunov stable in the norm (5.5) with $C_{3}$ replaced by $K / a$ when (6.11) or equivalently (6.12) holds, where $\lambda$ and $\mu_{0}(0)$ are defined by (4.7) and (4.12) and $M_{-}(\cdot)$ is defined by (5.20). In (6.12), $\mu_{0}(0)$ may be replaced by $\mu_{1}$ as defined by (5.19). Under these conditions, the disturbance energy $\mathscr{E}[\psi]$, disturbance potential enstrophy $\mathscr{Z}[\psi]$, and disturbance available potential energy on the horizontal boundaries $\mathscr{P}_{i}[\psi]$, are bounded for all time in terms of the initial disturbance fields according to (6.1), (6.8) and (6.9).

\section{Recovery of some previous results}

The following special cases all concern the standard quasi-geostrophic model, for which $B=0$.

\subsection{Homogeneous boundary conditions}

If the basic state has uniform $\Theta_{i}$ on both horizontal surfaces, then a restricted problem may be considered by assuming the disturbance to have the homogeneous boundary conditions

$$
\theta_{i}=0 \quad(i=1,2), \quad \oint_{\partial D_{j}} \psi_{n} \mathrm{~d} s=0 \quad \text { on } \partial D_{j} \quad(j=1, \ldots, J) .
$$

By (2.3b) and (2.4), these conditions are dynamically self-consistent: if they hold for the initial disturbance, then they continue to hold for all time. It follows that $\mathscr{P}_{i}[\psi]=0$.

For such a basic state the functions $\Psi_{i}^{\alpha}(\xi)$ for $i=1,2$ are undefined, but we may still define the function $\Psi_{3}^{\alpha}(\xi ; z)$ according to $(3.1 a)$, and we consider basic states satisfying 
(3.2a). Taking (3.7) except with $G_{i}(\xi)=0$, the disturbance pseudoenergy/momentum (3.6) becomes

$$
\mathscr{A}=\mathscr{E}[\psi]+\iiint_{\Omega}\left\{\rho \int_{Q}^{Q+\iota}\left[\Psi_{3}^{\alpha}(\xi ; z)-\Psi_{3}^{x}(Q ; z)\right] \mathrm{d} \xi\right\} \mathrm{d} x \mathrm{~d} y \mathrm{~d} z .
$$

Using (4.1) and (7.2), we obtain

$$
C_{3} \mathscr{Z}[\psi] \leqslant \mathscr{E}[\psi]-\mathscr{A}
$$

Now, because of the homogeneous lateral boundary conditions, $\psi$ satisfies (4.8) note that $B=0$ here - and there is no need to partition the disturbance fields as in (4.3). Furthermore, because of the homogeneous boundary conditions in the vertical, the eigenvalue problem (4.13)-(4.14) is replaced by the usual vertical structure equation (cf. Pedlosky 1987, \$6.12) for which the minimum eigenvalue is $\mu=0$ : this corresponds to the barotropic mode. Thus (4.16) applies with $K=0$ and with $\psi^{\prime}$ replaced by $\psi$, and in place of (4.19) we obtain the Poincaré inequality

$$
\mathscr{E}[\psi] \leqslant \frac{\mathscr{Z}[\psi]}{\lambda}
$$

Combining (7.3) with (7.4) yields

$$
\left(C_{3}-\frac{1}{\lambda}\right) \mathscr{Z}[\psi] \leqslant-\mathscr{A}
$$

Whenever

$$
C_{3} \hat{\lambda}>1
$$

we see from (7.5) that $\mathscr{A} \leqslant 0$; then $\mathscr{Z}[\psi]$ and $\mathscr{E}[\psi]$ are bounded for all time in terms of the positive invariant $(-\mathscr{A})$ according to

$$
\mathscr{Z}[\psi] \leqslant \frac{\lambda(-\mathscr{A})}{C_{3} \lambda-1}, \quad \mathscr{E}[\psi] \leqslant \frac{(-\mathscr{A})}{C_{3} \lambda-1} .
$$

This establishes nonlinear stability for basic states satisfying (7.6), recovering the result of McIntyre \& Shepherd (1987, Appendix B). When (5.4b) holds, Liapunov stability can be established.

\subsection{Eady's problem}

Eady's problem of baroclinic instability is a classical one in geophysical fluid dynamics (e.g. Pedlosky $1987, \S 7.7$ ). In addition to $B=0$, we take $\beta=0, \rho=$ const., $N^{2}=$ const., and $\eta=0$, and consider a domain consisting of a periodic zonal channel of width $\Delta y$. Let $\Delta z \equiv z_{2}-z_{1}$. Eady's basic state is given by

$$
\Psi=-s y z, \quad Q=f, \quad \Theta_{i}=-s y,
$$

which represents a zonal basic flow with constant vertical shear $s$. Because the basic state has uniform potential vorticity, the analysis of $\$ 6$ is relevant to this problem. It is easily seen that the functions $\Psi_{i}^{\alpha}(\xi)$ are given by

$$
\Psi_{i}^{\alpha}(\xi)=\left(z_{i}-\frac{\alpha}{s}\right) \xi ;
$$

if we choose $\alpha=s\left(z_{1}+z_{2}\right) / 2$, then we may take

$$
C_{1}=C_{2}=\frac{1}{2} \Delta z
$$

in $(3.2 b)$. 
By Theorem 6.1, Eady's basic state (7.8) is nonlinearly stable whenever (6.11) is satisfied. To evaluate $M_{-}(-\lambda)$, note that the functions $U(z, \mu)$ and $V(z, \mu)$ in $(5.20)$ are in this case given by

$$
U(z, \mu)=\cos \left((\mu S)^{1 / 2}\left(z-z_{1}\right)\right), \quad V(z, \mu)=\frac{\sin \left((\mu S)^{1 / 2}\left(z-z_{1}\right)\right)}{(\mu S)^{1 / 2}} .
$$

It follows that

$$
M_{-}(-\lambda)=\frac{(\lambda S)^{1 / 2} \Delta z}{2 \sinh \left((\lambda S)^{1 / 2} \Delta z\right)}\left(\cosh \left((\lambda S)^{1 / 2} \Delta z\right)-1\right),
$$

and the stability condition (6.11) holds if and only if

$$
\left(\frac{(\lambda S)^{1 / 2} \Delta z}{2}\right) \tanh \left(\frac{(\lambda S)^{1 / 2} \Delta z}{2}\right)>1
$$

For a periodic zonal channel of infinitely long extent and width $\Delta y, \lambda=(\pi / \Delta y)^{2}$. If $w_{0}$ is the constant satisfying $\left(w_{0} / 2\right) \tanh \left(w_{0} / 2\right)=1\left(w_{0} \approx 2.3994\right.$, numerically), then the condition (7.13) may be equivalently written as

$$
\frac{N \pi \Delta z}{f \Delta y}>w_{0}
$$

This recovers the recent result of Liu \& Mu (1996), though now Liapunov stability is also established. Since violation of the condition (7.14) is also precisely the condition for linear normal-mode instability (Pedlosky 1987, \$7.7), in this case the stability condition is both sufficient and necessary: therefore, it is the best possible.

\subsection{The generalized Charney-Stern theorem}

Consider a zonally symmetric basic state satisfying either

$$
\infty>c_{3}^{+} \geqslant Q_{y} \geqslant c_{3}^{-}>0, \quad \infty>c_{i}^{+} \geqslant(-1)^{i+1} \Theta_{i y} \geqslant c_{i}^{-}>0 \quad(i=1,2),
$$

or

$$
\infty>c_{3}^{+} \geqslant-Q_{y} \geqslant c_{3}^{-}>0, \quad \infty>c_{i}^{+} \geqslant(-1)^{i} \Theta_{i y} \geqslant c_{i}^{-}>0 \quad(i=1,2),
$$

for some positive constants $c_{i}^{+}$and $c_{i}^{-}(i=1,2,3)$. In the case of (7.15), we may take the limit $\alpha \rightarrow-\infty$ in which case (3.1) and (3.2) apply with $C_{i} \rightarrow|\alpha| / c_{i}^{+} \rightarrow \infty$. In the case of (7.16), this last expression holds in the limit $\alpha \rightarrow \infty$. In either case, the contribution of $\mathscr{E}[\psi]$ to $\mathscr{A}$ is asymptotically negligible and we obtain the inequalities

$$
\frac{\mathscr{Z}[\psi]}{c_{3}^{+}}+\sum_{i=1}^{2} \frac{\mathscr{P}_{i}[\psi]}{c_{i}^{+}} \leqslant \frac{(-\mathscr{A})}{|\alpha|} \leqslant \frac{\mathscr{Z}[\psi]}{c_{3}^{-}}+\sum_{i=1}^{2} \frac{\mathscr{P}_{i}[\psi]}{c_{i}^{-}} .
$$

Conservation of $\mathscr{A}$ in time, together with (7.17) and either (7.15) or (7.16), then yields

$$
\frac{\mathscr{Z}[\psi]}{c_{3}^{-}}+\sum_{i=1}^{2} \frac{\mathscr{P}_{i}[\psi]}{c_{i}^{-}} \leqslant \max \left\{\frac{c_{1}^{+}}{c_{1}^{-}}, \frac{c_{2}^{+}}{c_{2}^{-}}, \frac{c_{3}^{+}}{c_{3}^{-}}\right\}\left(\frac{\mathscr{Z}\left[\psi_{0}\right]}{c_{3}^{-}}+\sum_{i=1}^{2} \frac{\mathscr{P}_{i}\left[\psi_{0}\right]}{c_{i}^{-}}\right) .
$$

This establishes nonlinear Liapunov stability in the norm defined by the square root of the left-hand side of (7.18) - recovering the finite-amplitude Charney-Stern theorem of Shepherd (1989). 


\section{An example}

We consider the standard quasi-geostrophic model with constant $N^{2}$ and constant density scale height $H$, in a periodic zonal channel $0 \leqslant x \leqslant 2 \pi L, 0 \leqslant y \leqslant Y$, with $z_{1}=0, z_{2}=2 H$, and with topography $\eta=h \sin (\pi y / Y)[1+\cos (x / L)], h$ being a positive constant. Consider a steady basic state satisfying

$$
\begin{gathered}
\Psi=-C_{3}(Q-f)=-C_{3}\left(\nabla^{2} \Psi+\frac{\Psi_{z z}}{S}-\frac{\Psi_{z}}{S H}+\beta y\right) \quad \forall z \\
\Psi=-C \Theta=-C\left(\Psi_{z}+f S \eta\right) \quad \text { at } \quad z=0 \\
\Psi=C \Theta=C \Psi_{z} \quad \text { at } \quad z=2 H
\end{gathered}
$$

with $\Psi_{x}=0$ at $y=0, Y$.

We must first establish that such a basic state exists. Let

$$
\Psi(x, y, z)=y \phi_{1}(z)+\sin (\pi y / Y) \phi_{2}(z)+\cos (x / L) \sin (\pi y / Y) \phi_{3}(z) .
$$

Substituting (8.2) into (8.1), we find that $\phi_{i}(z)(i=1,2,3)$ are the solutions of the following boundary-value problems:

$$
\frac{\phi_{i}^{\prime \prime}}{S}-\frac{\phi_{i}^{\prime}}{S H}+\lambda_{i} \phi_{i}=a_{i},\left.\quad\left(\phi_{i}^{\prime}+\frac{\phi_{i}}{C}\right)\right|_{z=0}=b_{i},\left.\quad\left(\phi_{i}^{\prime}-\frac{\phi_{i}}{C}\right)\right|_{z=2 H}=0,
$$

where $a_{1}=-\beta, a_{2}=0=a_{3}, b_{1}=0, b_{2}=-f S h=b_{3}$, and

$$
\lambda_{1}=\frac{1}{C_{3}}, \quad \lambda_{2}=\frac{1}{C_{3}}-\frac{\pi^{2}}{Y^{2}}, \quad \lambda_{3}=\frac{1}{C_{3}}-\frac{\pi^{2}}{Y^{2}}-\frac{1}{L^{2}} .
$$

We know that a non-trivial solution to (8.3) exists if the corresponding homogeneous problem has only the trivial solution. But the homogeneous version of (8.3) is identical to (4.13) and (4.14) with $K=1$, after making the appropriate choice of $\mu$. It follows that the smallest non-trivial eigenvalue of $(8.3)$ is $\mu_{0}(1)$, which is identical to $m_{0}$ in (5.25). For this geometry, $\lambda=(\pi / Y)^{2}$. Therefore, provided the basic state satisfies (5.25), we have $\lambda_{2}<m_{0}$ and $\lambda_{3}<m_{0}$ by hypothesis and the homogeneous version of (8.3) has only the trivial solution for $i=2,3$. It thus remains to consider $i=1$. Now, $\lambda_{1}$ is a positive eigenvalue of the homogeneous version of (8.3) if and only if (5.18) holds with $K=1$ and $\mu=\lambda_{1}$. Taking $B_{i}=0$ and $C_{1}=C_{2}=C$ in this case, together with $K=1$ and $\mu=\lambda_{1}$, and using (B 1) and (B 2), (5.18) reduces to

$$
\ell_{3} \operatorname{coth} \ell_{1}=\frac{H}{C}-\frac{C}{4 H}\left(1-\ell_{1}^{2}\right)
$$

where $\ell_{1} \equiv\left(1-4 \lambda_{1} H^{2} S\right)^{1 / 2}$. (If $\lambda_{1}>1 / 4 H^{2} S$, then $\ell_{1}$ is imaginary and (8.5) may be more conveniently rewritten as

$$
\tilde{\ell}_{1} \cot \tilde{\ell}_{1}=\frac{H}{C}-\frac{C}{4 H}\left(1+\tilde{\ell}_{1}^{2}\right)
$$

where $\tilde{\ell}_{1} \equiv\left(4 \lambda_{1} H^{2} S-1\right)^{1 / 2}$.) However, for a given $\lambda_{1}$, (8.5) will only hold for one value of $C>0$. Thus it is certainly possible to find a basic state satisfying (8.1).

Using (B 3) with $C_{1}=C_{2}=C, B=0$, and $Z=1$, any basic state satisfying (8.1) is nonlinearly stable by Theorem 5.1 provided that

$$
M_{-}\left(\frac{1}{C_{3}}-\lambda\right) \equiv \frac{C}{2 H}\left\{\frac{\ell \cosh \ell-\left(\ell^{2}+\sinh ^{2} \ell\right)^{1 / 2}}{\sinh \ell}\right\}>1
$$


and

where $\ell \equiv\left(1+4\left[\lambda-\left(1 / C_{3}\right)\right] H^{2} S\right)^{1 / 2}$.

$$
C_{3} \lambda>1
$$

It is instructive to compare these stability conditions with those of $\mathrm{Mu} \&$ Simon (1993). For this example, Mu \& Simon's stability condition (23) takes the form

$$
\lambda>\frac{3 \mathrm{e}^{2}}{C S H}+\frac{4}{C^{2} S}+\frac{1}{C_{3}} \text {. }
$$

Evidently (8.7) requires $(8.6 b)$, so the interesting comparison is between $(8.6 a)$ and (8.7). Given $C_{3}$ and $\lambda$ satisfying (8.6b), (8.6a) implies stability for all

$$
C>C_{\mathrm{LMS}} \equiv \frac{2 H \sinh \ell}{\ell \cosh \ell-\left(\ell^{2}+\sinh ^{2} \ell\right)^{1 / 2}},
$$

while (8.7) implies stability for all

$$
C>C_{\mathrm{MS}} \equiv \frac{4 H}{\ell^{2}-1}\left(\frac{3}{2} \mathrm{e}^{2}+\left((9 / 4) \mathrm{e}^{4}+4\left(\ell^{2}-1\right)\right)^{1 / 2}\right) .
$$

It may be verified that the ratio $C_{\mathrm{MS}} / C_{\mathrm{LMS}}$ approaches $3 \mathrm{e}^{2} \tanh 1 \approx 16.88$ as $\ell \rightarrow 1^{+}$ (note that $(6.4 b)$ requires $\ell>1$ ), and decreases as $\ell$ increases, approaching the asymptotic limit of 4 as $\ell \rightarrow \infty$. Thus the range of stable flows allowed by (8.8) is considerably larger than that allowed by (8.9).

\section{Discussion}

We have considered continuously stratified quasi-geostrophic flow in a domain $D \times\left[z_{1}, z_{2}\right]$, where the horizontal domain $D$ is multiply connected. Both the standard quasi-geostrophic model and the modified quasi-geostrophic model of White (1977) have been treated. Steady basic states satisfying $(3.2 a, b)$ and $(5.4 a, b)$ have been shown to be nonlinearly Liapunov stable (Theorem 5.1) whenever the condition (5.24) (equivalently (5.25)) is satisfied. For steady basic states with horizontally uniform potential vorticity, satisfying $(3.2 b)$ and $(5.4 b)$, the corresponding condition (Theorem 6.1) is (6.11) (equivalently (6.12)). In both cases, the theorems are analogues of Arnol'd's (1966) second stability theorem, and depend on the establishment of the Poincare inequality (4.19); this inequality has been shown to be optimal, in the sense that equality can be realized. For practical applications, Liapunov stability is less important than the fact that certain important disturbance norms can be uniformly bounded for all time in terms of the initial disturbance fields, with the bounds going to zero uniformly as the initial disturbance goes to zero. The explicit bounds are provided by (5.1)-(5.3) in the case of Theorem 5.1, and by (6.1), (6.8) and (6.9) in the case of Theorem 6.1 .

The basic states under consideration are, in general, required only to be steady. When, however, the governing equations are zonally symmetric (which requires that the topography $\eta_{i}$ and the lateral boundaries be independent of $x$ ), we have restricted attention to zonally symmetric basic states. We now justify this restriction by showing that when the dynamics are zonally symmetric, only zonally symmetric basic states can satisfy our stability theorems. We do this by contradiction, in the spirit of Andrews (1984). Suppose that there exists a basic state $\left(\Psi, Q, \Theta_{i}\right)$ that is provably stable by Theorem 5.1 or Theorem 6.1 , and is not zonally symmetric. We can then consider a non-zero disturbance $\left(\psi, q, \theta_{i}\right)$ generated by a zonal translation of this 
basic state. By the zonal symmetry of the problem, this disturbance does not alter any of the conserved quantities $\mathscr{E}, \mathscr{M}$ or $\mathscr{C}$; hence $\mathscr{A}$ defined by (3.6) must vanish. On the other hand, $\psi$ satisfies (4.8), and hence the Poincare inequality (4.19) holds for $\psi$. Since $K>1$ by hypothesis, this implies that $\mathscr{E}[\psi]<\mathscr{W}^{2}$. (In the case of Theorem 6.1 , note that $\mathscr{Z}[\psi]=0$ for this translational disturbance.) On the other hand, from (4.27) or (6.5) - noting that $\overline{\mathscr{W}}_{0}=0$, and that $\mathscr{Y}=0$ in the latter case we have $\mathscr{A} \leqslant \mathscr{E}[\psi]-\mathscr{W}^{2}$. Taken together these inequalities imply $\mathscr{A}<0$, which is a contradiction.

Since the Poincare inequality (4.19) used in the present study is optimal, it is clear that no improvement on our stability criteria is possible using the present approach, and our results are in that sense definitive. Yet the criteria are only sufficient for stability, not necessary; one is naturally led to wonder what happens when they are violated. Vanneste (1995) has recently shown that, in the case of basic flows with zero horizontal shear, the multilayer quasi-geostrophic stability theorem of $\mathrm{Mu}$ et al. (1994), when taken together with the usual Arnol'd first theorem, is both necessary and sufficient for stability: when its criteria are violated, the basic flow is either linearly unstable in a normal-mode sense, or is unstable to a nonlinear explosive resonant instability. It would be interesting to see whether a similar result could be obtained in the present case of continuously stratified flow.

L.Y. is supported by the State Education Commission of China and LASG. M.M. is supported by the National Natural Science Foundation of China and LASG. T.G.S. is supported by the Natural Sciences and Engineering Research Council and the Atmospheric Environment Service of Canada. This paper is the result of collaboration undertaken while L.Y. and M.M. were visiting the University of Toronto.

\section{Appendix A. Proof of (2.5)}

We consider both the modified quasi-geostrophic model with $B \neq 0$, and the standard model with $B=0$. Integrating (2.1) over $D$, and using (2.2) and (2.3), yields

$$
\iint_{D} \Phi_{z t} \mathrm{~d} x \mathrm{~d} y=\frac{f_{1}(t)}{r(z)}
$$

where $f_{1}(t)$ is some function of $t$ alone.

We first show that $f_{1}(t)=0$. If $B=0$, then applying (A 1) at either horizontal surface in conjunction with (2.4) implies $f_{1}(t)=0$. If $B \neq 0$, we may integrate (A 1$)$ in $z$ to obtain

$$
\begin{aligned}
\left.\iint_{D} \Phi_{i t} \mathrm{~d} x \mathrm{~d} y\right|_{i=1} ^{i=2} & =\frac{f_{1}(t)}{f^{2}} \int_{z_{1}}^{z_{2}} \frac{N^{2}}{\rho} \mathrm{d} z=-\frac{f_{1}(t)}{f^{2}} \int_{z_{1}}^{z_{2}}\left(\frac{g \rho_{z}}{\rho^{2}}+\frac{g^{2}}{\rho c^{2}}\right) \mathrm{d} z \\
& =\frac{g f_{1}(t)}{f^{2}}\left(\frac{1}{\rho_{2}}-\frac{1}{\rho_{1}}\right)-\frac{g^{2} f_{1}(t)}{f^{2}} \int_{z_{1}}^{z_{2}} \frac{\mathrm{d} z}{\rho c^{2}} .
\end{aligned}
$$

Then (2.4) and (A 2) imply

$$
\left.\frac{1}{B_{i}} \iint_{D} \Phi_{z i t} \mathrm{~d} x \mathrm{~d} y\right|_{i=1} ^{i=2}=\left.\iint_{D} \Phi_{i t} \mathrm{~d} x \mathrm{~d} y\right|_{i=1} ^{i=2}=\frac{g f_{1}(t)}{f^{2}}\left(\frac{1}{\rho_{2}}-\frac{1}{\rho_{1}}\right)-\frac{g^{2} f_{1}(t)}{f^{2}} \int_{z_{\mathrm{i}}}^{z_{2}} \frac{\mathrm{d} z}{\rho c^{2}}
$$


On the other hand, applying (A 1) at both horizontal surfaces yields

$$
\left.\frac{1}{B_{i}} \iint_{D} \Phi_{z i t} \mathrm{~d} x \mathrm{~d} y\right|_{i=1} ^{i=2}=\frac{g f_{1}(t)}{f^{2}}\left(\frac{1}{\rho_{2}}-\frac{1}{\rho_{1}}\right) .
$$

Taken together, (A 3) and (A 4) imply $f_{1}(t)=0$.

Since $f_{1}(t)=0$, it follows from (A 1$)$ that

$$
\iint_{D} \Phi_{t} \mathrm{~d} x \mathrm{~d} y=f_{2}(t)
$$

where $f_{2}(t)$ is some function of $t$ alone. We now show that $f_{2}(t)=0$. If $B=0$, then $\Phi$ is undetermined to within a function of $t$ and we may simply set $f_{2}(t)=0$ without loss of generality. If $B \neq 0$, then applying (A 5) with (A 1) at either horizontal surface in conjunction with (2.4) implies $f_{2}(t)=0$.

In both cases, therefore, (2.5) follows.

\section{Appendix B. Expression for $M_{-}(\mu)$}

In the special case $N^{2}=$ const. and $\rho_{0}(z)=\rho_{0}(0) \mathrm{e}^{-z / H}$ with $H=$ const., an explicit expression for the function $M_{-}(\cdot)$ can be obtained. In this case, $U(z, \mu)$ and $V(z, \mu)$ are given by

$$
\begin{gathered}
U(z, \mu)=\exp \left(\frac{z-z_{1}}{2 H}\right)\left\{\cosh \left(\frac{\ell\left(z-z_{1}\right)}{2 H}\right)-\frac{1}{\ell} \sinh \left(\frac{\ell\left(z-z_{1}\right)}{2 H}\right)\right\}, \\
V(z, \mu)=\frac{2 H}{\ell} \exp \left(\frac{z-z_{1}}{2 H}\right) \sinh \left(\frac{\ell\left(z-z_{1}\right)}{2 H}\right),
\end{gathered}
$$

where $\ell \equiv\left(1-4 \mu H^{2} S\right)^{1 / 2}$. Then from $(5.20)$,

$$
\begin{aligned}
M_{-}(\mu)= & \frac{\ell}{4 H}\left(C_{1}+C_{2}\right) \operatorname{coth}(\ell Z)+\left(C_{1}-C_{2}\right)\left(\frac{B}{2}-\frac{1}{4 H}\right) \\
& -\frac{\ell}{4 H}\left\{\left[\left(C_{1}-C_{2}\right) \operatorname{coth}(\ell Z)+\left(C_{1}+C_{2}\right)\left(\frac{2 H B}{\ell}-\frac{1}{\ell}\right)\right]^{2}+\frac{4 C_{1} C_{2}}{\sinh ^{2}(\ell Z)}\right\}^{1 / 2},
\end{aligned}
$$

where $Z \equiv\left(z_{2}-z_{1}\right) / 2 H$ and $B \equiv B_{1}=B_{2}$.

\section{REFERENCES}

ANDREws, D. G. 1984 On the existence of nonzonal flows satisfying sufficient conditions for stability. Geophys. Astrophys. Fluid Dyn. 28, 243-256.

ARNOL'D, V. I. 1966 On an a priori estimate in the theory of hydrodynamical stability. Izv. Vyssh. Uchebn. Zaved. Matematika 54(5), 3-5. (English transl. Am. Math. Soc. Transl., Series 2 79, 267-269 (1969).)

Bowman, J. C. \& SHEPHERD, T. G. 1995 Nonlinear symmetric stability of planetary atmospheres. $J$. Fluid Mech. 296, 391-407.

Cho, H.-R., Shepherd, T. G. \& Vladimirov, V. A. 1993 Application of the direct Liapunov method to the problem of symmetric stability in the atmosphere. J. Atmos. Sci. 50, 822-836.

FJøRTofT, R. 1950 Application of integral theorems in deriving criteria of stability for laminar flows and for the baroclinic circular vortex. Geofys. Publ. 17(6), 1-52.

Holm, D. D., Marsden, J. E., Ratiu, T. \& Weinstein, A. 1985 Nonlinear stability of fluid and plasma equilibria. Phys. Rep. 123, 1-116.

KuSHNER, P. J. \& SHEPHERD, T. G. 1995 Wave-activity conservation laws and stability theorems for semi-geostrophic dynamics. Part 2. Pseudoenergy-based theory. J. Fluid Mech. 290, 105-129. 
LiU YONGMing \& MU Mu 1996 Nonlinear stability theorem for Eady's model of quasigeostrophic baroclinic flow. J. Atmos. Sci. 53, 1459-1463.

MCINTYRe, M. E. \& Shepherd, T. G. 1987 An exact local conservation theorem for finite-amplitude disturbances to non-parallel shear flows, with remarks on Hamiltonian structure and on Arnol'd's stability theorems. J. Fluid Mech. 181, 527-565.

Mu Mu \& Shepherd, T. G. 1994 On Arnol'd's second nonlinear stability theorem for twodimensional quasi-geostrophic flow. Geophys. Astrophys. Fluid Dyn. 75, 21-37.

Mu Mu \& Simon, J. 1993 A remark on nonlinear stability of three-dimensional quasigeostrophic motions. Chin. Sci. Bull. 38, 1978-1984.

MU MU \& WANG XIYONG 1992 Nonlinear stability criteria for the motion of three-dimensional quasigeostrophic flow on a $\beta$-plane. Nonlinearity $5,353-371$.

Mu MU \& ZeNG QINGCUN 1991 Criteria for the nonlinear stability of three-dimensional quasigeostrophic motions. Adv. Atmos. Sci. 8, 1-10.

Mu Mu, Zeng Qingcun, Shepherd, T. G. \& Liu Yongming 1994 Nonlinear stability of multilayer quasi-geostrophic flow. J. Fluid Mech. 264, 165-184.

Pedlosky, J. 1987 Geophysical Fluid Dynamics, 2nd edn. Springer.

Shepherd, T. G. 1988 a Nonlinear saturation of baroclinic instability. Part I: The two-layer model. J. Atmos. Sci. 45, 2014-2025.

SHEPHERD, T. G. $1988 b$ Rigorous bounds on the nonlinear saturation of instabilities to parallel shear flows. J. Fluid Mech. 196, 291-322.

SHEPHERD, T. G. 1989 Nonlinear saturation of baroclinic instability. Part II: Continuously stratified fluid. J. Atmos. Sci. 46, 888-907.

ShePherd, T. G. 1990 Symmetries, conservation laws, and Hamiltonian structure in geophysical fluid dynamics. Adv. Geophys. 32, 287-338.

Swaters, G. E. 1986 A nonlinear stability theorem for baroclinic quasigeostrophic flow. Phys. Fluids 29, 5-6.

Szeri, A. \& Holmes, P. 1988 Nonlinear stability of axisymmetric swirling flows. Phil. Trans. R. Soc. Lond. A 326, 327-354.

VANNESTE, J. 1995 Explosive resonant interaction of baroclinic Rossby waves and stability of multilayer quasi-geostrophic flow. J. Fluid Mech. 291, 83-107.

WHITE, A. A. 1977 Modified quasi-geostrophic equations using geometric height as vertical coordinate. Q. J. R. Met. Soc. 103, 383-396. 